\title{
Excited state quantum phase transitions and chaos in the Dicke model
}

\author{
P. Pérez-Fernández*, A. Relaño ${ }^{\dagger}$, P. Cejnar**, J. M. Arias ${ }^{\ddagger}$, J. Dukelsky ${ }^{\S}$ \\ and J. E. García-Ramos ${ }^{\text {II }}$ \\ *Departamento de Física Aplicada III, Escuela de Ingenieros, Universidad de Sevilla, ES-41092 \\ Sevilla, Spain \\ ${ }^{\dagger}$ Departamento de Física Aplicada I and GISC, Universidad Complutense de Madrid, Av. \\ Complutense s/n, 28040 Madrid, Spain \\ ** Institute of Particle and Nuclear Physics, Faculty of Mathematics and Physics, Charles \\ University, V Holešovičkách 2, Prague, 18000, Czech Republic \\ ${ }^{*}$ Departamento de Física Atómica, Molecular y Nuclear, Facultad de Física, Universidad de \\ Sevilla, 41080 Sevilla, Spain \\ ${ }^{\S}$ Instituto de Estructura de la Materia, IEM-CSIC, Serrano 123, E-28006 Madrid, Spain \\ "Departamento de Física Aplicada, Universidad de Huelva, 21071 Huelva, Spain
}

\begin{abstract}
In this contribution, the critical behavior of excited state quantum phase transitions (ESQPT's) and its relation to order and chaos in the Dicke model are studied. This model is nonintegrable. For comparison, the same problem is studied for the Jaynes-Cummings model which is, somehow, the integrable version of the Dicke model. The existence of an ESQPT is confirmed in both cases. However, in the Dicke model the signatures of criticality in excited states are blurred by the onset of quantum chaos. It is concluded that the emergence of quantum chaos is caused by the precursors of the excited state quantum phase transition.
\end{abstract}

Keywords: Quantum chaos, excited state quantum phase transition

PACS: 05.45.Mt, 05.30.Rt, 05.70.Fh, 42.50.Nn

\section{INTRODUCTION}

One of the goals of many-body physics is the understanding of quantum critical phenomena [1]. Most of the studies up to now have been done for the ground state quantum phase transition (QPT). This is a sudden change of the ground state-structure at a certain critical value of a control parameter. It can be observed as a nonanalytic evolution of the system's energy and wave function induced by an adiabatic variation of the control parameter, let's call it $\lambda$, across the quantum critical point at zero temperature. Depending on the physical phenomenon to be focused, different Hamiltonians have been studied. Usually these depend on a single control parameter which drives the system from one phase to the other.

Very recently, a new quantum critical phenomenon, related with excited states rather than to the ground state, has been discovered [2]. This is called excited state quantum phase transition (ESQPT). Similarly to the ground state case, an ESQPT is defined as a nonanalytical evolution of individual excited states with the control parameter. It can also be observed as a singular variation of the state density with energy and entails dramatic dynamical consequences [3, 4]. 
So far, such effects have been studied mostly in integrable systems with one degree of freedom - systems characterized by regular dynamics. This is the case for the Lipkin model, for example. A question arises naturally, can ESQPT's be also found in more complex and chaotic systems? In relation to this question, if ESQPT's appear in chaotic systems where the level repulsion is essential, how is affected the collapse of quantum energy levels, typical of an ESQPT, by the level repulsion, inherent to quantum chaotic systems? We would like to give an answer to these questions by analyzing a simplified model of collective interactions of matter and light known as the Dicke model [5].

\section{THE MODELS}

The Dicke model describes an array of $N$ two-level atoms interacting with a photonic field. Thus, it models a composite system consisting of two interacting subsystems: i) a single bosonic mode given by creation and annihilation operators $b^{\dagger}$ and $b$ which is described by the Heisenberg-Weyl algebra HW(1), and ii) a subsystem represented by pseudospin operators $J_{ \pm}=J_{x} \pm i J_{y}$ and $J_{0}=J_{z}$ satisfying the commutation relations of the su(2) algebra. A useful realization of this algebra can be constructed with an array of spin-1/2 particles (two-level atoms) located on $2 J$ sites:

$$
\begin{array}{r}
J_{+}=\sum_{i=1}^{2 J} a_{\uparrow i}^{\dagger} a_{\downarrow i}, \quad J_{-}=\sum_{i=1}^{2 J} a_{\downarrow i}^{\dagger} a_{\uparrow i}, \\
J_{0}=\frac{1}{2} \sum_{i=1}^{2 J}\left(a_{\uparrow i}^{\dagger} a_{\uparrow i}-a_{\downarrow i}^{\dagger} a_{\downarrow i}\right) .
\end{array}
$$

$a_{\uparrow i}^{\dagger}\left(a_{\uparrow i}\right)$ and $a_{\downarrow i}^{\dagger}\left(a_{\downarrow i}\right)$ create (annihilate) spin-up and spin-down states of the fermion on site $i$, and the ladder operators $J_{ \pm}$describe spin flips along the array. The total pseudospin length is $J=N_{a} / 2$, where $N_{a}$ is the total number of atoms. The system has two degrees of freedom associated with $N_{b}$ (number of photons) and $J_{z}$. The Dicke Hamiltonian [5]

$$
H_{1}(\lambda)=\omega_{0} J_{z}+\omega b^{\dagger} b+\frac{\lambda}{\sqrt{4 J}}\left[\left(b+b^{\dagger}\right)\left(J_{+}+J_{-}\right)\right]
$$

conserves the parity $\Pi=(-1)^{M / 2}$ labeling individual eigenstates, being $M=2\left(N_{b}+\right.$ $\left.J_{z}+J\right)$. This model is not integrable. The Hilbert space dimension is infinite since we can have any number of photons. From a practical point of view this means that the photon space needs to be truncated by a certain maximal value $N_{b}$. In the numerical calculations we checked that an increase of $N_{b}^{\max }$ did not cause changes in results.

If we perform the called rotating-wave approximation in $H_{1}$, we get a simpler Hamiltonian $[6,7]$ :

$$
H_{2}(\lambda)=\omega_{0} J_{z}+\omega b^{\dagger} b+\frac{\lambda}{\sqrt{4 J}}\left[b J_{+}+b^{\dagger} J_{-}\right] .
$$

This is the Jaynes-Cummings Hamiltonian which conserves $M$. The conservation of this quantity implies a finite dimension of the Hilbert space. This model is integrable. 


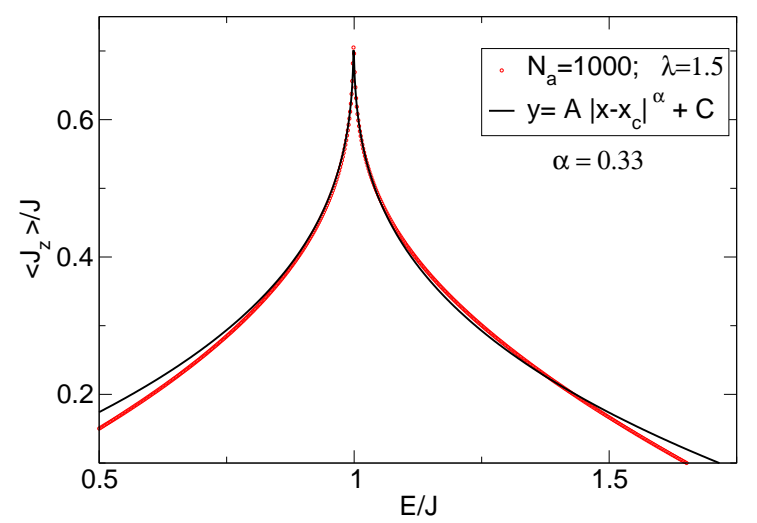

FIGURE 1. (color online) Scaled atomic inversion as a function of energy for the Jaynes-Cummings model. Calculations are done for $\omega_{0}=1, \omega=2, \lambda=1.5$, and $J=500$.

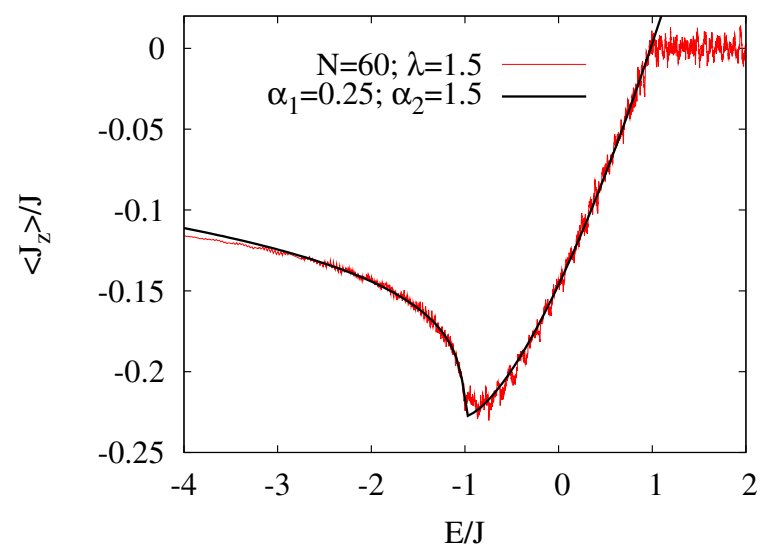

FIGURE 2. (color online) Scaled atomic inversion as a function of energy for the Dicke model. Calculations done for $\omega_{0}=\omega=1, \lambda=1.5, J=30$ and results smoothed over 20 points.

\section{RESULTS}

Both Hamiltonians present a QPT of second order $[8,9,10]$ in the thermodynamical limit, $J \rightarrow \infty$ and their properties have been obtained from a semiclassical analysis [11]. For $\lambda>\lambda_{c}\left(\lambda_{c}\right.$ is the value of the control parameter for which the system undergoes the QPT) the QPT in both models is followed by a chain of excited-state phase transitions located at the critical energy, which corresponds approximately with the ground-state energy of the normal phase.

We investigate the singular dependence of the order parameter $\left\langle J_{z}\right\rangle$ close to the critical energy $E_{c}$. The dependence has the form

$$
\left\langle J_{z}\right\rangle=\left\langle J_{z}\right\rangle_{c}+A\left|E-E_{c}\right|^{\alpha},
$$

which is characterized by a critical exponent $\alpha$. In Fig. 1 we show results for the Jaynes-Cummings model with 1000 atoms for $\lambda=1.5$. The red points correspond to the expectation value of $J_{z}$ in each eigenstate with eigenvalue $E$. The continuous black 
curve shows a fit with Eq. (4) to the numerical results for $\left\langle J_{z}\right\rangle$. We get a critical exponent $\alpha=0.33$ and observe a cusp singularity at $E_{c} / J=1$, which correspond to the value of the critical energy of the ESQPT.

We find similar results for the Dicke model. Fig. 2 shows a calculation with 60 atoms and $\lambda=1.5$. The thin red oscillating line represents numerical data for $\left\langle J_{z}\right\rangle$ obtained as an average over 20 eigenstates around the eigenvalue $E$. The full black line is the fit of those values with Eq. (4). We get different critical right and left exponents at the value of the critical energy of the ESQPT, which in this case is $E_{c} / J=-1$. A comparison between the two figures shows that the results for the Dicke model are fuzzier than those for the Jaynes-Cummings model [12].

It is well known that the Dicke model is nonintegrable and partly chaotic. The superradiant transition at zero temperature was shown $[8,9,10]$ to be correlated with a crossover from ordered to chaotic behavior. Therefore we can ask whether or not quantum chaos is related to the critical behavior of excited states. Chaotic properties in the spectrum introduce large fluctuations that partly hide the singular dependence at the critical point in the Fig. 2. However, chaos and ESQPT have some properties which are difficult to conciliate. On the one hand, the most significant feature of quantum chaos is the level repulsion, which entails a null probability of finding two levels at the same energy. On the other hand, an ESQPT is typically connected with a rather close approach of levels, often with a singular accumulation of levels at the critical energy. Therefore, the relation between the level repulsion and spectral signatures of an ESQPT constitutes a interesting theoretical challenge.

In order to analyze the relationship between chaos and ESQPT we compute the spacing distribution $P(s)$, where $s$ is a normalized $(\bar{s}=1)$ distance between two neighboring levels, on both sides of the critical energy $E_{c}$. It is well known that if the distribution follows a Poissonian form, $P^{\mathrm{P}}=e^{-s}$, then the system is regular but if the distribution follows a Wigner form, $P^{\mathrm{W}}=\frac{\pi}{2} s e^{-\pi s^{2} / 4}$, then the system is chaotic [13]. We present results for $\lambda=3$, which is large enough to guarantee a large number of levels bellow the critical energy.

In Fig. 3 we show the $P(s)$ distributions calculated for both subcritical $\left(E<E_{c}\right)$ and supercritical $\left(E>E_{c}\right)$ parts of the spectrum and for various atom numbers. In all cases, we used the same number of energy levels for building the histograms on both sides of the critical energy. The most relevant fact is that bellow $E_{c}$ we observe $P(s=0)>0$, while above $E_{c}$ we obtain $P(s=0) \approx 0$. In other words, the levels start to repel each other when crossing the ESQPT critical energy, that is, the most significant signature of quantum chaos - level repulsion - is only present above the critical energy.

In order to obtain a more quantitative description, we analyze how the spectral statistics changes as one moves in the spectrum. For this purpose we use the accumulated spacing distribution $F(s)=\int_{0}^{s} d x P(x)$ and we define the following quantity:

$$
\Delta=\frac{\sum_{i}\left[F^{\mathrm{W}}\left(s_{i}\right)-F\left(s_{i}\right)\right]^{2}}{\sum_{i}\left[F^{\mathrm{W}}\left(s_{i}\right)-F^{\mathrm{P}}\left(s_{i}\right)\right]^{2}},
$$

where $F^{\mathrm{W}}$ and $F^{\mathrm{P}}$ are accumulated distributions derived from $P^{\mathrm{W}}$ and $P^{\mathrm{P}}$, respectively. This expression means that given a numerical sequence of spacings $\left\{s_{i}\right\}$, we measure its 


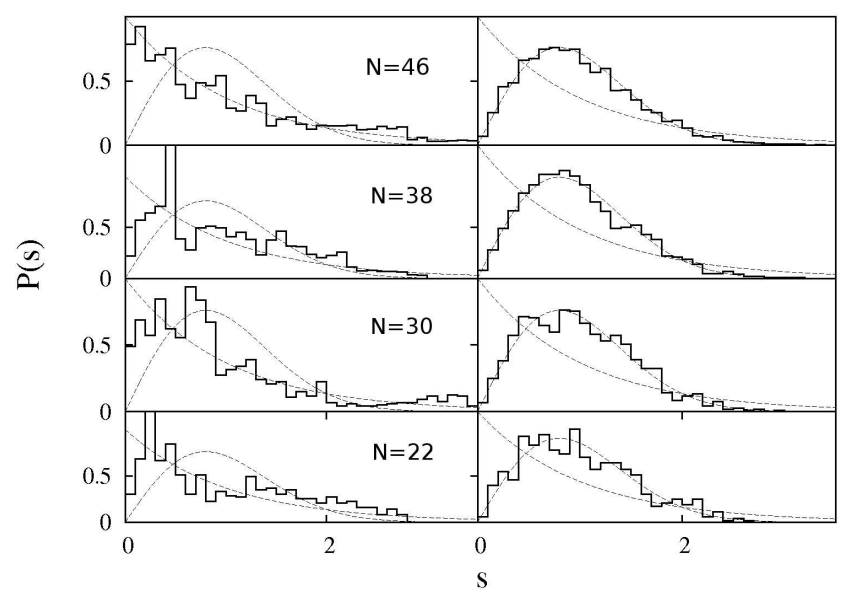

FIGURE 3. Nearest-neighbor spacing distribution $P(s)$ for the Dicke model with $\lambda=3$ and different values of $N$ (number of atoms). The left column shows the histograms for $E<E_{c}$, the right column for $E>E_{c}$.

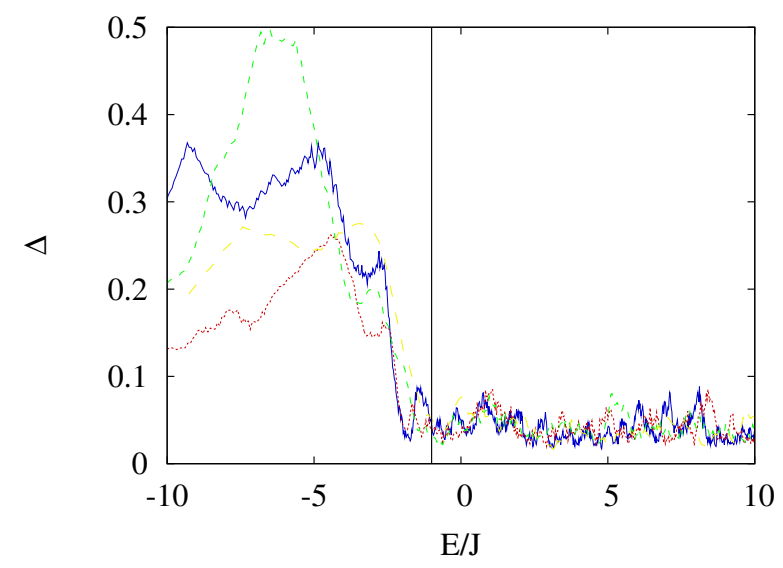

FIGURE 4. (color online) Onset of chaos in the Dicke model with $\lambda=3$ measured by the distance (5) of actual level statistics from the Wigner distribution. The distance is plotted as a function of the mean scaled energy of the relevant fraction of the spectrum, the vertical line indicating the critical energy. Calculation done for various atom numbers: $N=22$ (very light long-dashed line, yellow online), 30 (light medium-dashed line, green online), 38 (dark short-dashed line, red online), and 46 (very dark solid line, blue online).

"distance" from the Wigner surmise. Therefore, $\Delta=0$ if the numerical sequence follows the Wigner distribution, while $\Delta=1$ for a Poissonian sequence.

In Fig. 4 we can observe that a quite abrupt transition from finite values of $\Delta$ to $\Delta \sim 0$ takes place just below the critical energy $E_{c}$. Though the range of the covered values of $N$ is not large enough to approach the thermodynamic limit, it suffices to conjecture that the critical behavior in the order parameter is accompanied by a change in the spectral statistics. Furthermore, as this change takes place very abruptly, we postulate that it is caused by the precursors of the ESQPT. 


\section{CONCLUSION}

In conclusion, we have demonstrated the existence of an ESQPT in the Dicke model as well as in the Jaynes-Cummings model. The Dicke model exhibits a similar type of ESQPT, but the signatures are blurred by the onset of chaotic behavior in the spectrum. On the other hand, our numerical calculations show that a crossover, from the regime with no level repulsion to the one with the Wigner level statistics, takes place around the critical energy. These results are compatible with the hypothesis that this abrupt emergence of level repulsion is caused by the precursors of the ESQPT's. We anticipate the existence of a similar qualitative behavior in other nonintegrable systems with ESQPT's.

\section{ACKNOWLEDGEMENTS}

This work is presented on the occasion of Franco Iachello's 70th birthday. This work has been partially supported by the Spanish Ministerio de Ciencia e Innovación and FEDER funds under projects FIS2011-28738-c02-01. Spanish Consolider Ingenio Programme CPAN - CSD2007-00042 and by Junta de Andalucía FQM-160 and P07-FQM-02894.

\section{REFERENCES}

1. S. Sachdev, Quantum Phase Transitions, Cambridge University Press, Cambridge, 1999.

2. $\quad$ M. A. Caprio, P. Cejnar, and F. Iachello, Ann. Phys. 323, 1106-1135 (2008).

3. A. Relaño, J. M. Arias, J. Dukelsky, J. E. García-Ramos, and P. Pérez-Fernández, Phys. Rev. A 78, 060102(R) (2008).

4. P. Pérez-Fernández, A. Relaño, J. M. Arias, J. Dukelsky, and J. E. García-Ramos, Phys. Rev. A 80, 032111 (2009).

5. R. H. Dicke, Phys. Rev. 93, 99 (1954).

6. E. T. Jaynes, and F. W. Cummings, Proc. IEEE 51, 81 (1963).

7. M. Tavis, and E. T. Jaynes, Phys. Rev. 170, 379 (1968).

8. C. Emary, and T. Brandes, Phys. Rev. E 67, 066203 (2003).

9. C. Emary, and T. Brandes, Phys. Rev. Lett. 90, 044101 (2003).

10. N. Lambert, C. Emary, and T. Brandes, Phys. Rev. Lett. 92, 073602 (2004).

11. P. Pérez-Fernández, P. Cejnar, J. M. Arias, J. Dukelsky, J. E. García-Ramos, and A. Relaño, Phys. Rev. A 83 (2011).

12. P. Pérez-Fernández, A. Relaño, J. M. Arias, P. Cejnar, J. Dukelsky, and J. E. García-Ramos, Phys. Rev. E 83 (2011).

13. H.-J. Stöckmann, Quantum Chaos: An Introduction, Cambridge University Press, Cambridge, UK, 1999. 